\title{
CONTROL MPPT APLICADO A SISTEMAS GIRASOL PARA LA MÁXIMA EFICIENCIA
}

\section{MPPT CONTROL SYSTEMS USED IN SUNFLOWER FOR MAXIMUM EFFICIENCY}

\author{
MS(c). Diego Armando Mejía Bugallo, PhD. Ivaldo Torres Chávez. \\ MSc. Jorge Luis Díaz Rodríguez.
}

Universidad de Pamplona, Facultad de Ingenierías y Arquitectura. Ciudadela Universitaria. Pamplona, Norte de Santander, Colombia. Tel.: (+577) 568 5303, Fax: (+577) 568 5303, Ext. 144.

E-mail: damejia14@hotmail.com, \{ivaldot, jdiazcu@gmail.com\}

\begin{abstract}
Resumen: En este artículo se presenta el desarrollo de un sistema solar tipo girasol con un sistema de seguimiento de máximo punto de potencia autoajustable (MPPT), el cual consta de un seguidor solar con paneles fotovoltaicos y un sistema de control MPPT que permite conocer las curvas (I-V), (P-V). La implementación del sistema Girasol con el seguimiento del punto de máxima potencia de los paneles solares se construyó a escala real con el objetivo de suministrar energía limpia a los cubículos de profesores. Los análisis basados en las pruebas de investigación y del desarrollo, confirman que el sistema de girasol aumenta la eficiencia con la implementación del sistema MPPT, aumentado la captura de radiación solar y la producción de energía eléctrica en comparación a los sistemas tradicionales fotovoltaicos fijos.
\end{abstract}

Palabras clave: Sistemas fotovoltaico, panel solar, eficiencia, máximo punto de potencia autoajustable (MPPT).

\begin{abstract}
This paper deals with the development of a complete solar system, which consists of a solar tracker with photovoltaic panels, called Sunflower, and a system of MPPT control, which plays with the curves (I-V), (P-V) presents features photovoltaic panels. The main content of this development is focused on the operating principles of Sunflower and in monitoring the maximum power point of solar panels; this development was made to scale, to provide light to a Building. The experiment focuses on achieving the optimization of the energy from a PV system via two methodologies. The first is to increase the amount of solar radiation received by the panel. The second is to improve the internal components of a photovoltaic panel so that its yield increases. Analysis and evidence-based research and development, confirm that the sunflower increases the capture of solar radiation and this is more efficient than fixed PV systems. And it has further improved systemefficiency with MPPT.
\end{abstract}

Keywords: Electric, photovoltaic system, sunflower system, energy optimization.

\section{INTRODUCCIÓN}

En el Annual Energy Outlook 2015 (AEO2015) desarrollado en Washington, DC, predice que la distribución mundial de la demanda de energía va a cambiar más profundamente, con un uso de la energía básicamente estable en gran parte de Europa, Japón, Corea y Norteamérica, y un consumo creciente concentrado en el resto de Asia (el 60\% del total mundial), África, Oriente Medio y América Latina (Awerbuch, 2004). 
Actualmente la tecnología de las energías renovables todavía se encuentra en una etapa de desarrollo $^{1}$. En particular para la energía solar fotovoltaica, el desarrollo se centra principalmente en mejorar su eficiencia (Luque y Hegedus, 2003), por medio de dos fases, una mejorando la captura de la radiación solar y otra realizando el seguimiento del punto de máxima potencia de los paneles solares. Garantizando un almacenamiento adecuado de la energía producida.

La situación actual de Colombia frente al cambio climático, el fenómeno del niño, pone en riesgo la generación y distribución de energía a todos los ciudadanos (UNESCO, 2008). Por ende nace esta alternativa de energía renovable ya que el modelo energético actual, a base de combustibles fósiles como principal fuente, genera un gran impacto ambiental y no es sustentable.

\section{PROCESO EXPERIMENTAL Y TEORÍA}

Los sistemas fotovoltaicos están conformados básicamente por paneles solares, reguladores de tensión, baterías e invasores DC/AC tal y como se muestra en la figura 1 .

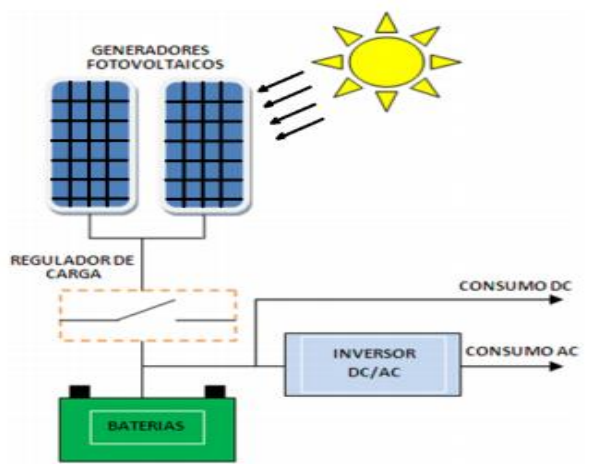

Fig. 1. Sistema fotovoltaico integrado porpaneles, inversores, baterías y reguladores de tensión.

Uno de las características importantes en los sistemas fotovoltaicos es la de mantener el suministro de energía eléctrica a la carga asociada todo el tiempo, e incluso en horas que no hay luz solar ${ }^{4}$. Para esto existen diversas estrategias que han permitido mejorar la eficiencia del sistema fotovoltaico sin tener que sobredimensionarlo.

Una de las estrategias utilizadas en los sistemas fotovoltaicos es la de utilizar elementos que sean capaces de seguir la trayectoria solar para lograr el aumento en la captación de la radiación solar durante el día; entre los cuales se encuentran (Esram y Chapman, 2007), (Meinel y Meinel, 2004).
Algunas metodologías para mejorar la captación de radiación solar son:

- Estructura de soporte estático. Es el sistema más habitual que se encuentra en las instalaciones y su configuración se ubica el sistema dependiendo de la latitud y longitud del lugar teniendo en cuenta la inclinación más adecuada para captar la mayor radiación solar posible (Meinel y Meinel, 2004).

- Sistemas de seguimiento solar de 1 eje. En esta configuración el soporte realiza un seguimiento solar. La rotación del soporte se hace por medio de un solo eje, bien sea horizontal, vertical u oblicuo. Este tipo de seguimiento es el más sencillo y el más económico resultando sin embargo incompleto ya que sólo podrá seguir o la inclinación o el máximo zenit del Sol, pero no ambas a la vez (Meinel y Meinel, 2004).

- Sistemas de seguimiento solar de dos ejes. Con este sistema es posible obtener de forma continua una posición perpendicular de la estructura con respecto a la radiación solar durante el día, consiguiendo una mayor captación de esta. gracias a este sistema es posible realizar un seguimiento total del sol en altitud $y$ en Zenit (Meinel y Meinel, 2004).

En este trabajo se habla del aumento de la eficiencia de un sistema fotovoltaico por medio de dos fases. Una es utilizando el posicionamiento autónomo siguiendo la intensidad del brillo solar, y la segunda fase es realizando el seguimiento del punto de máxima potencia en los paneles solares. El sistema de seguidor solar consta de dos ejes y se ubica en el plano del eje $X$ y el eje $Z$ logrando mantener la máxima incidencia solar en los paneles solares de forma perpendicular, como se muestra en la figura 2. Esto aumenta la captura de radiación solar, permitiendo aumentar la eficiencia en comparación con los sistemas solares estáticos (Beleño et al., 2011).

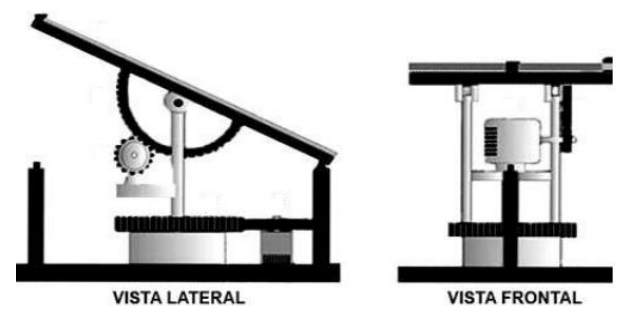

Fig. 2. Sistema seguidor solar. 
En cuanto a la segunda fase, consta de un control MPPT, el cual es conformado por un convertidor DC/DC en configuración SEPIC, y un algoritmo de control el cual se encarga de realizar el seguimiento del punto máximo de potencia en los paneles solares, como puede verse en la figura 3.

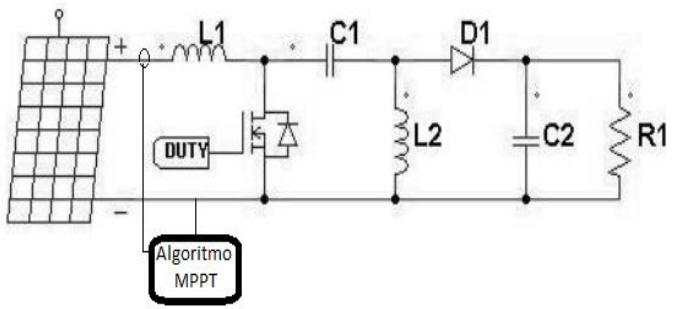

Fig. 3. Sistema SEPIC para el control MPPT.

\section{PROCESO EXPERIMENTAL DEL GIRASOL SOLAR}

Para el desarrollo del proceso experimental del girasol solar, se diseñó una estructura metálica la cual es capaz de sostenes 2 paneles solares de 200 Watts, consta de dos grados de articulación permitiendo hacer el seguimiento solar completo y sele adecuo un sensor de intensidad lumínica, el cual trabaja en base a diferencias lumínicas.

Para la ubicación del sistema fotovoltaico, se adecuo un sensor, el cual consta de 4 fotoceldas en forma de cruz, separadas por una estructura plástica, estructura la cual crea el diferencial de luminosidad cuando no se está en forma perpendicular al sol, ver figura 4 .

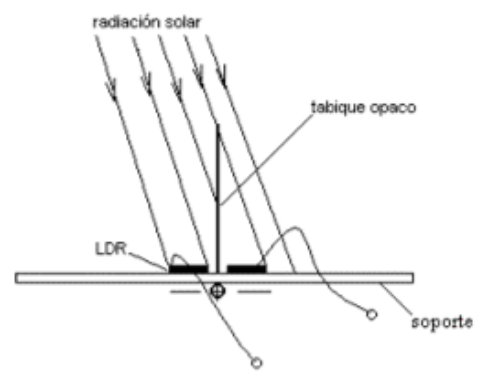

Fig. 4. Sensor de luminosidad.

Cada par de fotoceldas generan la acción de control para el respectivo grado de libertad, y se ejecuta por un circuito electrónico y de potencia, ver figura 5 .

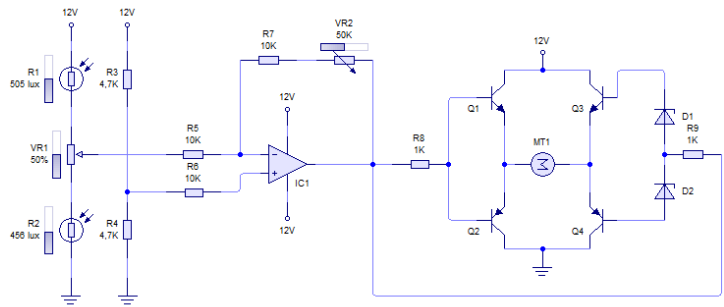

Fig. 5. Circuito de control para cada grado de libertad.

\section{PROCESO EXPERIMENTAL DEL SISTEMA MPPT}

En el desarrollo del control MPPT, se desarrolló un circuito SEPIC, con la ejecución de diferentes tipos de algoritmos entre estos $\mathrm{P} \& \mathrm{O}$, conductancia incremental, voltaje constante.

El circuito SEPIC, tiene la posibilidad de elevar, mantener o disminuir el nivel de tensión a la salida del convertidor, $\sin$ invertir la polaridad a la salida. El análisis se llevó a cabo con tres algoritmos diferentes.

\subsection{Análisis del método de voltaje constante}

Consiste en mantener el modulo en un valor de referencia de tensión (que corresponde a la tensión de salida en el punto de máxima potencia). Por esto, para una temperatura constante, el punto de máxima potencia se mueve horizontalmente; es decir, para cualquier valor de la radiación, el voltaje que asegura la máxima transferencia de potencia es prácticamente el mismo y está delimitado dentro de un pequeño rango. Sin embargo cuando hay variaciones de temperatura en la celda, la tensión MPP cambia considerablemente, pues ya no coincide con el valor de referencia cambiando el punto de operación de MPP.

En la implementación analógica de este método, la tensión del módulo es leído por un sensor de voltaje y se compara con la tensión de referencia, ver figura 6. De esta comparación resulta el error, el cual pasa por el modulador PWM, generando el ciclo de trabajo que activará el convertidor de conmutación. 


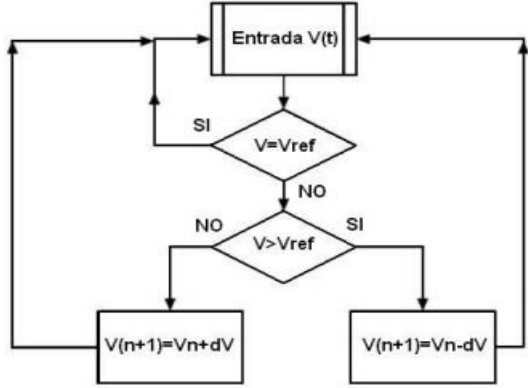

Fig. 6. Diagrama de flujo del método voltaje constante.

\subsection{Análisis del método Perturbación y Observación (P\&O)}

Es el método más empleado, por su sencillez y facilidad de implementación. En general, el proceso consiste en medir los valores de voltaje y corriente del panel para calcular la potencia que está entregando. Este valor de potencia se compara con el valor calculado anterior mente y se toma la decisión respecto al aumento o disminución del voltaje de referencia, Ver figura 7.

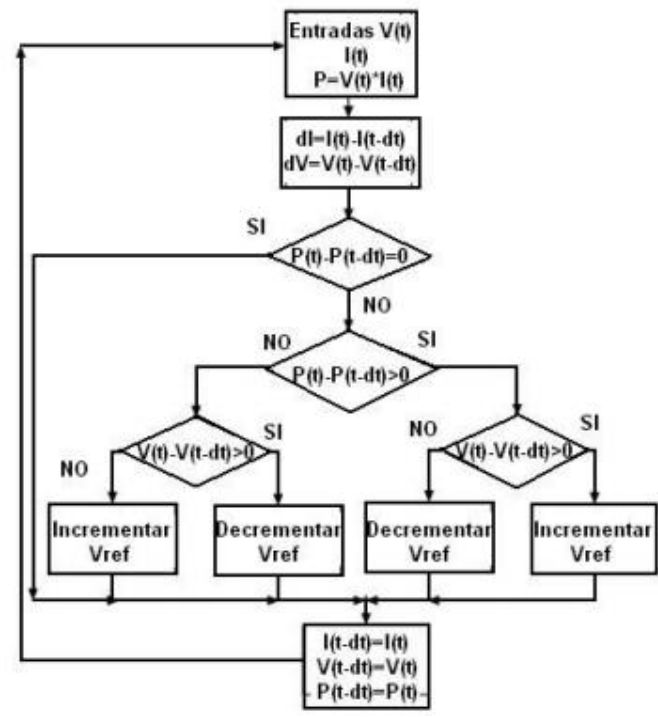

Fig. 7. Diagrama de flujo del método $P \& O$ diseñado para este trabajo.

A pesar de ser más compleja que la técnica anterior, $\mathrm{P} \& \mathrm{O}$ tiene problemas de parpadeo alrededor del punto de funcionamiento. Cuando la perturbación es grande el sistema alcanza el régimen con mayor velocidad, pero con las oscilaciones de tensión más altas en todo el punto óptimo. No obstante, cuando este paso es pequeño, el sistema se vuelve más lento y proporciona una mayor variación en el voltaje del módulo alrededor del MPP.

\subsection{Análisis del método conductancia incremental}

Este algoritmo es capaz de detenerse cuando detecta el punto máximo de potencia, de este modo se eliminan las oscilaciones alrededor de dicho punto, ver figura 8 .

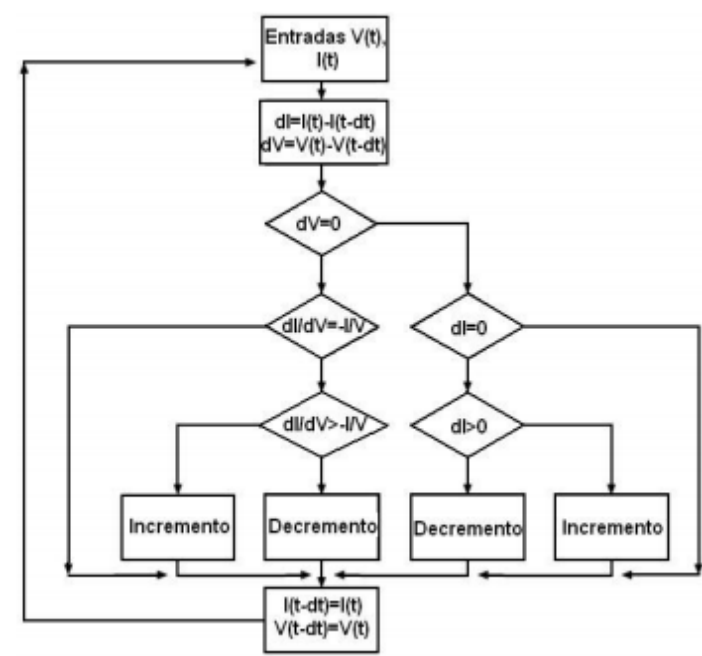

Fig. 8. Diagrama de flujo del método conductancia incremental.

\section{RESULTADOS}

Los resultados obtenidos, se lograron comparando las salidas de potencia del sistema de paneles, bajo una temperatura ambiente, y una radiación solar constante, en sistemas fotovoltaicos fijos y sistema el sistema tipo girasol.

El sistema fotovoltaico consta de 2 paneles fotovoltaicos de 80 Watts, 20 Voltios, 4 Amp. Se le conectó a la salida de estos una carga de $50 \Omega$. Obteniendo los siguientes resultados:

Tabla 1. Comparación de potencias en sistemas fijo y tipo girasol.

\begin{tabular}{|c|c|c|}
\hline Hora & $\begin{array}{c}\text { Potencia de } \\
\text { sistema fijo }\end{array}$ & $\begin{array}{c}\text { Potencia de } \\
\text { sistema girasol }\end{array}$ \\
\hline $6: 00$ & $0 \mathrm{~W}$ & $2.4 \mathrm{~W}$ \\
\hline $7: 00$ & $6 \mathrm{~W}$ & $10 \mathrm{~W}$ \\
\hline $8: 00$ & $20 \mathrm{~W}$ & $25 \mathrm{~W}$ \\
\hline $9: 00$ & $45 \mathrm{~W}$ & $51 \mathrm{~W}$ \\
\hline $10: 00$ & $80 \mathrm{~W}$ & $95 \mathrm{~W}$ \\
\hline $11: 00$ & $129.4 \mathrm{~W}$ & $133 \mathrm{~W}$ \\
\hline $12: 00$ & $140 \mathrm{~W}$ & $146 \mathrm{~W}$ \\
\hline $13: 00$ & $132 \mathrm{~W}$ & $136 \mathrm{~W}$ \\
\hline $14: 00$ & $82 \mathrm{~W}$ & $100 \mathrm{~W}$ \\
\hline $15: 00$ & $50 \mathrm{~W}$ & $63 \mathrm{~W}$ \\
\hline $16: 00$ & $16 \mathrm{~W}$ & $20 \mathrm{~W}$ \\
\hline $17: 00$ & $8.6 \mathrm{~W}$ & $14 \mathrm{~W}$ \\
\hline $18: 00$ & $0 \mathrm{~W}$ & $1.3 \mathrm{~W}$ \\
\hline
\end{tabular}


A continuación se observa la figura 9, con los resultados gráficos de comparación de potencias entre los dos tipos de sistemas.

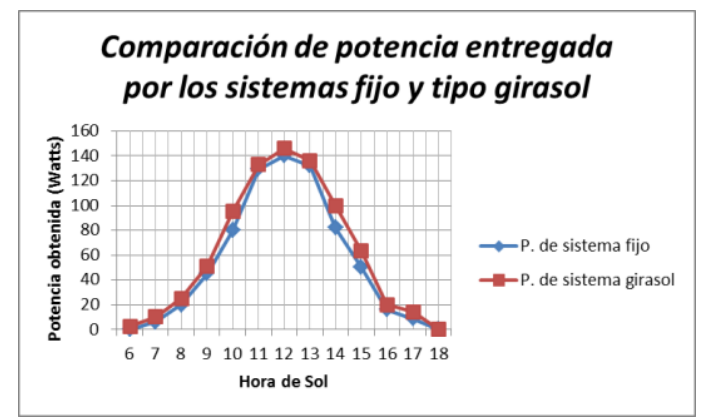

Fig. 9. Comparación de potencia entregada por los sistemas fijo y tipo girasol.

Revisando solo los resultados del sistema tipo girasol, se observa un incremento notorio en la generación de potencia por parte del sistema fotovoltaico.

Comparando la mejora de potencia con el sistema MPPT, se obtuvieron los siguientes resultados, ver figura 10:

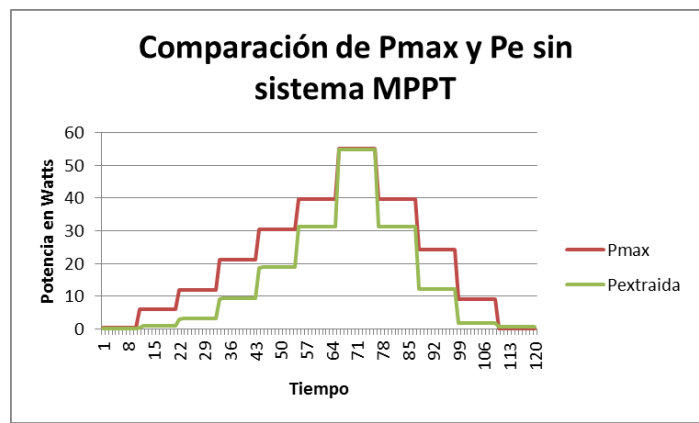

Fig. 10. Comparación de la potencia a obtenery de la potencia obtenida sin el sistema MPPT.

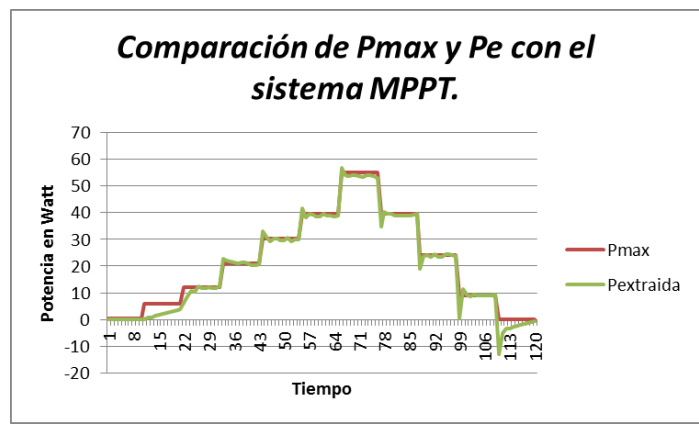

Fig. 11. Comparación de la potencia a obtenery de la potencia obtenida con el sistema MPPT.

Se observa que el sistema de control MPPT, extrae la máxima potencia del panel, de forma aceptable, incrementando aún más la potencia de salida del sistema fotovoltaico.

Por último se presenta la tabla 2 , en donde se compara la potencia de salida del sistema sin ningún tipo de control ni seguimiento solar, contra el sistema fotovoltaico con seguimiento solar y control MPPT.

Tabla 2. Comparación del sistema simple, el sistema controlado.

\begin{tabular}{|c|c|c|}
\hline Hora & $\begin{array}{c}\text { Potencia de } \\
\text { sistema simple }\end{array}$ & $\begin{array}{c}\text { Potencia de sistema } \\
\text { controlado }\end{array}$ \\
\hline $6: 00$ & $0 \mathrm{~W}$ & $3.6 \mathrm{~W}$ \\
\hline $7: 00$ & $6 \mathrm{~W}$ & $10 \mathrm{~W}$ \\
\hline $8: 00$ & $20 \mathrm{~W}$ & $33 \mathrm{~W}$ \\
\hline $9: 00$ & $45 \mathrm{~W}$ & $60 \mathrm{~W}$ \\
\hline $10: 00$ & $80 \mathrm{~W}$ & $115 \mathrm{~W}$ \\
\hline $11: 00$ & $129.4 \mathrm{~W}$ & $145 \mathrm{~W}$ \\
\hline $12: 00$ & $140 \mathrm{~W}$ & $160 \mathrm{~W}$ \\
\hline $13: 00$ & $132 \mathrm{~W}$ & $151 \mathrm{~W}$ \\
\hline $14: 00$ & $82 \mathrm{~W}$ & $118 \mathrm{~W}$ \\
\hline $15: 00$ & $50 \mathrm{~W}$ & $63 \mathrm{~W}$ \\
\hline $16: 00$ & $16 \mathrm{~W}$ & $27 \mathrm{~W}$ \\
\hline $17: 00$ & $8.6 \mathrm{~W}$ & $14 \mathrm{~W}$ \\
\hline $18: 00$ & $0 \mathrm{~W}$ & $3 \mathrm{~W}$ \\
\hline
\end{tabular}

A continuación se observan las curvas de potencias del sistema simple y del sistema controlado, ver figura 12. En donde se puede apreciar que el sistema que controla el seguimiento del sol y posee un control MPPT, es capaz de entregar una mayor potencia en iguales condiciones ambientales, en comparación al sistema fotovoltaico simple.

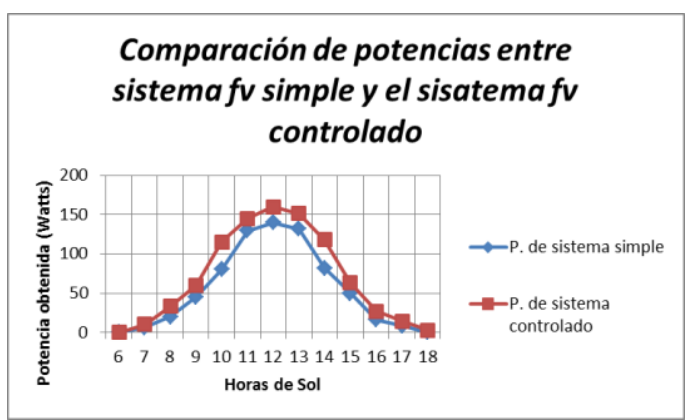

Fig.12. Comparación de potencias entre sistema fv simple y el sistema fu controlado.

\section{CONCLUS IONES}

Se determinó que para obtener un mayor rendimiento era necesario diseñar un sistema con 2 ejes de libertad, para lo cual se hizo uso de dos motores DC, los cuales están energizados a 12 voltios. 
Se logró una respuesta rápida del control de posición del panel solar al utilizar 4 sensores de luz LDRs, colocados de tal manera que cada 2 sensores sean ubicados de manera opuesta. De esta manera, se logró que de acuerdo al eje de referencia, 2 sensores guíen al sistema de derecha a izquierda y los otros 2 lo guíen de arriba abajo.

Con realizar simplemente el seguimiento solar, no basta para que nuestro sistema sea eficaz, se hace necesario utilizar las estrategias de control MPPT. La cual hace que los paneles solares entreguen su máxima potencia. Los sistemas con control MPPT, presentan una mayor eficiencia en comparación a los sistemas simples.

Se concluye que al unir estas dos estrategias de control (seguimiento solar y control MPPT), se obtiene una mayor potencia.

\section{RECONOCIMIENTOS}

El autor Diego Armando Mejía agradece el apoyo y acompañamiento por parte del grupo de investigación Logos y al Grupo de Investigación en Sistemas Energéticos de la Universidad de Pamplona.

\section{REFERENCIAS}

Awerbuch, S. (2004). Portfolio-Based Electricity Generation Planning: Implications for Renewables.

Luque, A., Hegedus, S. (2003). Handbook of Photovoltaic Science and Engineering. John Wiley \& Sons.

UNESCO (2008). El fenómeno meteorológico El Niño 1997-1998, a escala regional y por país es. Disponible en:

http://www.unesco.org.uy/phi/biblioteca/bitstr eam/123456789/468/1/el+niño+1998-2.pdf.

Esram T. y Chapman, P. L. (2007). "Comparison of photovoltaic array maximum power point tracking techniques", IEEE Trans. Energy Conversion, Vol. 22, No. 2, pp. 439-449, Jun.

Meinel, A. B. y Meinel, M. P. (2004). Aplicaciones de las energías solares, Editorial Reverte S.A.

Beleño, K., Torres, I., García, A. y Díaz, J. L. (2011). "Solar positioning system platform using Labview and the PIC18F4550 microcontroller". Revista Colombiana de Tecnologías de Avanzada, Vol. 2, No. 18. 